\title{
Chapter 2 \\ Classification of RI Investment Areas in South Africa
}

\subsection{Approaches to Research Infrastructure Investment}

The three approaches that are commonly used when assessing and identifying the need for investing and mainstreaming RI initiatives are the (i) bottom-up approach; (ii) top-down approach; and (iii) integrated approach (Khadka \& Vacik, 2012). The bottom-up approach allows for innovative ideas to be supported by subject experts as well as numerous other key role players, without any boundaries or parameters. This approach facilitates the articulation of a specific need that may not necessarily be identified or displayed on the radar screen of government departments or funding agencies (Girdwood, 2013). The bottom-up approach promotes co-creation of research programmes through the direct involvement, participation and consultation of various stakeholders. Considering the stronger uptake and ownership factor, this approach is widely adopted in most developed countries (Girdwood, 2013), where researchers tend to have a strong voice when presenting a case for funding to funding agencies and government departments. Unfortunately, in developing countries, due to competing investment priorities and constrained budgets, there is a long lead time associated with the bottom up approach as funding agencies and government departments may not have the capacity to fund additional research activities, let alone funding for the provisioning of RIs.

The top-down approach entails the development and adoption of science and technology policies that are driven from the highest level of the state, which in turn is able to ring-fence a budget (Khadka \& Vacik, 2012). Top-down policies demonstrate clear objectives and goals, hierarchy of authority, alignment to national imperatives, and resources to deliver on their implementation (Girdwood, 2013). However this approach may to some extent ignore the opinions and considerations of the research community. Consequently the implementation of many policies born from the top-down approach may be subject to scrutiny and failure (Girdwood, 2013). 


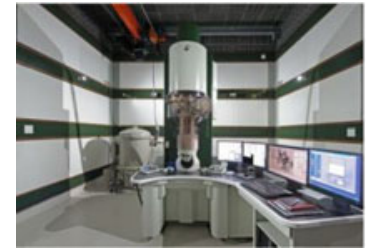

EM-ARM200F: Atomic Resolution Analytical Electron Microscope

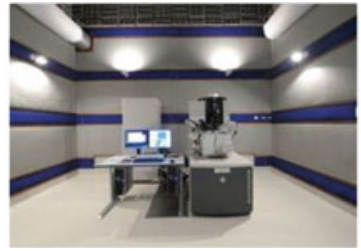

FEI HELIOS NANOLAB 650:

Focussed-ion beam Scanning Electron Microscope

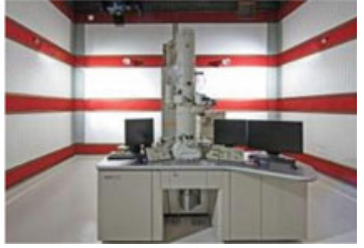

JEOL JEM 2100:

Analytical Transmission Electron Microscope

Fig. 2.1 Suite of high end microscopes available at the C-HRTEM at Nelson Mandela University, South Africa (Neethling, 2018)

Accelerated and impactful outcomes on RI investment require a dual approach to investments in RI, which integrates both the bottom-top and top-down approaches. This would, therefore, entail the development and adoption of science and technology policies on the one hand, driven from the highest level of the state and with dedicated ring-fenced budgets while on the other hand, the community is encouraged to participate and contribute towards policy formulation that addresses the requirements of the research community and societal needs (Girdwood, 2013). Such an approach tends to minimise the public demands for a short term return on investment from the usage of taxpayers' money and strengthens the impact of STI for societal benefit.

Case study In 1983, the research community, driven by the Microscopy Society of South Africa, spearheaded an initiative for the establishment of a Centre for High Resolution Transmission Electron Microscopy (HRTEM) in the country. Due to the uniqueness of the equipment as well as the high costs related to procuring, housing, maintaining and operating the equipment, this proposal by the research community was deemed high risk by government departments, including funding agencies, at the time. Once a champion was identified to drive this initiative, the proposal was reformulated as a business plan with a strategy that defined mitigation steps for managing potential risks. In 2009, 26 years after the project was first conceptualised, the first tranche of investment was secured for the establishment of a National Centre for HRTEM, which was ultimately launched in 2011. The suite of microscopes are presented in Fig. 2.1. Since the launch of the Centre, spanning 2011-2018, the following highlights have been reported (Neethling, 2018):

\section{Outputs from 2011 to 2018 :}

- Number of publications in accredited journals: 102

- Number of postgraduate students supported (Hons, M.Sc., Ph.D.): 253 
- Number of collaborations established with African and international partners: 20

- Number of national collaborations established: 20

- Number of private sector partners: 6

Research areas supported from 2011 to 2018:

- Strong materials, energy security, biotechnology, nanotechnology, catalysis, power plant steels, and nuclear materials.

\subsection{Process for Acquiring RI}

The process of motivating and securing a dedicated RI budget commences with a (i) needs assessment of RI; (ii) benchmarking of current RI against international developments; (iii) establishing and nurturing strong partnerships between stakeholders regionally, nationally and internationally; and (iv) clearly indicating the envisaged impact on the research landscape and society. The purpose of a needs assessment is to provide baseline information relating to the current state of (i) research infrastructure; and (ii) the human resources required to support such infrastructures. The process which is more complicated than it appears, can be guided by the following pertinent questions:

- What equipment is needed to support the national R\&D agenda?

- What is the current state of research equipment across the national research landscape? Is the equipment functional, in storage, decommissioned, broken, other?

- What is the age of the equipment across the national research landscape?

- What are the investments to date in research equipment?

- What is the spread of research equipment in terms of its geographical placement or location within the country?

- How does the placement of equipment support and/or advance research niche areas that align to the geographical position of the country globally?

- What is the quantity of skilled human resources available in the country to support, operate and maintain the research equipment?

- What are the qualifications and experience of the human resources that are available to support, operate and maintain the research equipment?

- What is required to create a critical mass of skilled human resources that can support, operate and maintain the research equipment?

- What is the spread of the human resources in terms of demographics such as age, gender and race?

- How does the country fare against similar countries (benchmarking) in terms of research infrastructure and human resources. 
- In which countries can collaborative networks be established for shared access to equipment and skills development?

This baseline and benchmarking groundwork sets the foundation for developing a framework for RI investments in a country, which in turn will feed into the development of a RI roadmap at a later stage. This framework can be further refined into a RI strategy which clearly defines RI investment categories, objectives, budgets and a timeframe within which specific deliverables or outcomes will be achieved.

The RI budget must support the development and/or acquisition of RIs that advance research in specific thematic areas that either explores and/or exploits the opportunities presented by the geographical positioning of the country globally. As an example, the geographical position of South Africa places it at a competitive advantage for research in areas such as (i) palaeontology; (ii) ocean currents; (iii) climate change; (iv) indigenous knowledge systems; (v) biodiversity; (vi) conservation; (vii) mining and minerals; and (viii) astronomy, amongst others (South African Department of Science and Technology, 2002).

Once the RI budget has been secured, a process must be developed that awards infrastructure grants on a competitive basis to public research performing institutions. The scientific case driving the justification or motivation for the infrastructure grant must advance the country's priority investment areas such as food security, clean water, energy security, health, poverty alleviation, amongst others. These areas in turn link to global programmes such as the SDGs and STISA 2024. Hence, a complementary, synergistic and integrated approach is required for mapping RIs, as outlined in Fig. 2.2.

\subsection{Classification of RIS in South Africa}

This section provides a model for mapping RI needs across the innovation value chain. Such a mapping exercise, was used to assess the RI needs across the innovation value chain in South Africa, comprising the integrated approach. This is described in detail in the draft research development and innovation funding framework that was launched by the Department of Science and Technology in 2010. Six major RI investment areas were identified and mapped against the four stages of the innovation value chain, i.e. (i) well-founded research laboratory equipment; (ii) scientific equipment; (iii) specialised facilities; (iv) high-end infrastructure; (v) access to global infrastructures; and (vi) cyber-infrastructure. A summary of the main RI categories is shown in Fig. 2.3. 


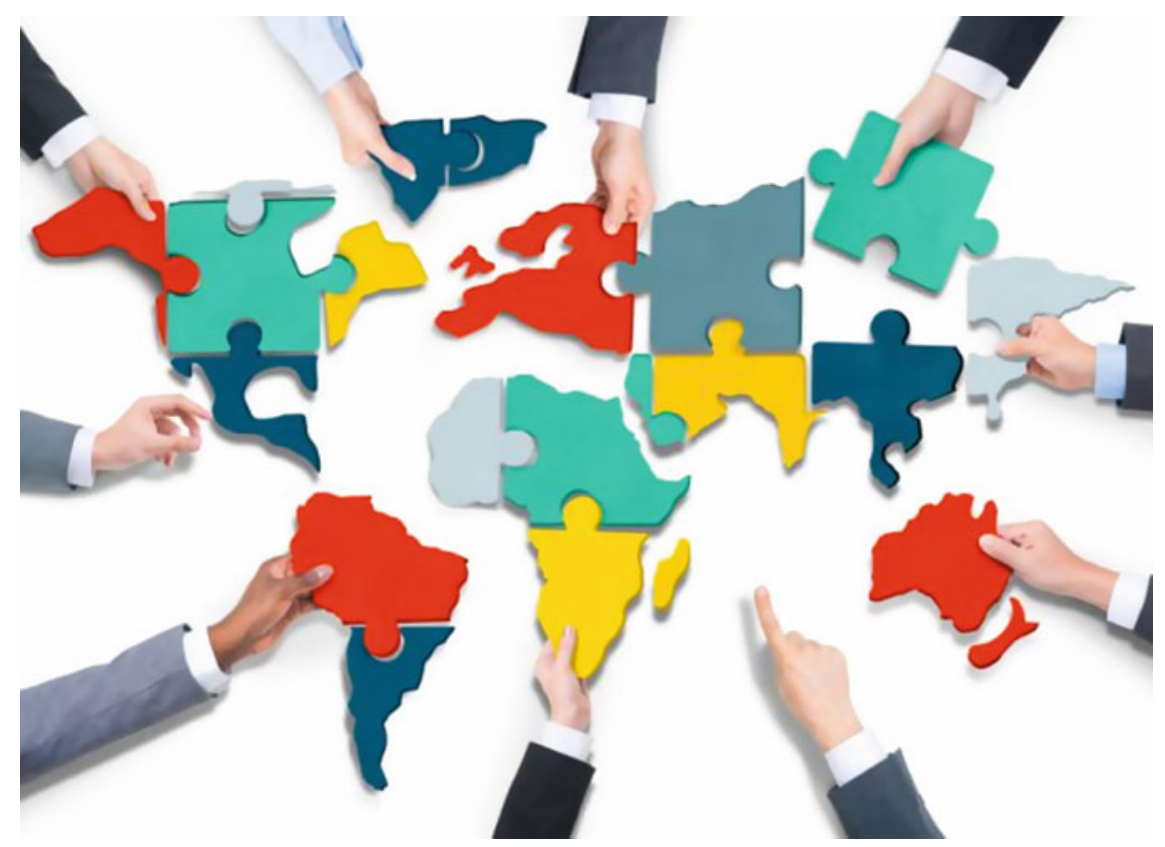

Fig. 2.2 A complementary, synergistic and integrated approach is required for mapping RIs

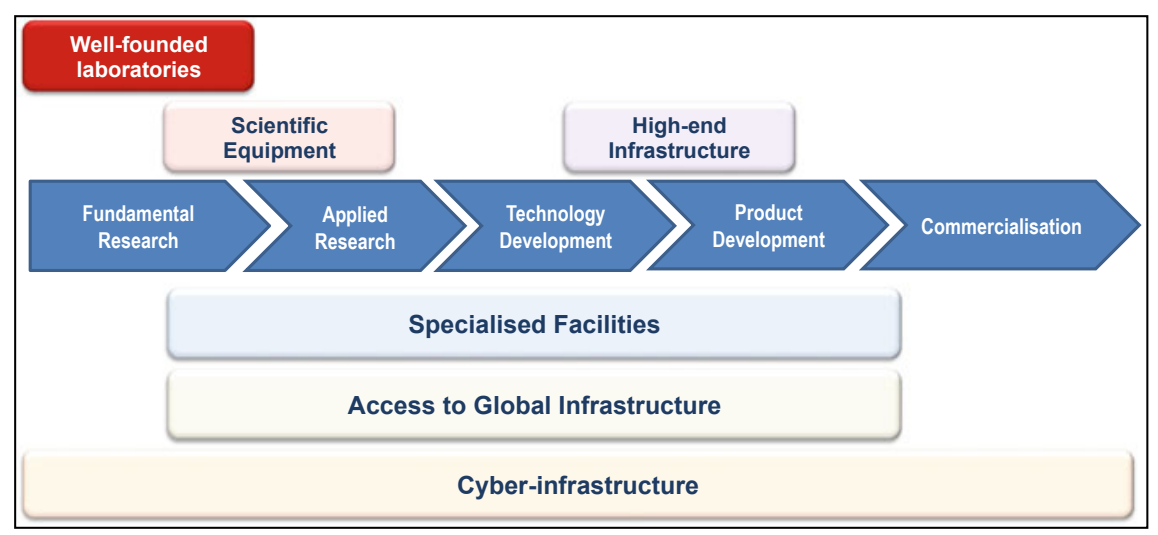

Fig. 2.3 Mapping the various RI funding categories across the innovation value chain

\subsubsection{Well-Founded Laboratory Research Equipment}

Well-founded laboratory research equipment includes the minimum level of equipment and facilities that need to be in place as a necessary requirement for conducting basic research and training postgraduate students. This sub-category of equipment 
refers to generally moveable or benchtop analytical or basic, entry-level instrumentation that is usually acquired and managed within a specific research group at a research institution. The full responsibility and costs associated with the equipment's operations, maintenance and access by other researchers or research groups is assumed by the research institution. Examples of this class of equipment include analytical NMR spectrometers; equipment for chromatography; and powder X-ray diffractometers. Well-founded laboratory equipment is usually a fundamental requirement for the functioning of any research and training laboratory, hence the funds required to support the procurement of such instruments must be sourced from either the research department or institution (South African Department of Science and Technology, 2010).

\subsubsection{Scientific Equipment}

Scientific equipment can be defined as those enabling research tools that are fundamental for conducting competitive research and training the next generation of researchers. Scientific equipment refers to dedicated, immovable, free standing, large, networked, multi-user and multi-disciplinary research equipment including all necessary ancillary components such as computers and specialised software, amongst others. In this case, resources need to be earmarked for constructing specialised buildings or other physical infrastructures for housing such equipment, in order to ensure that the optimal functional specifications of the equipment are met (South African Department of Science and Technology, 2010). Scientific equipment can be further divided into two sub-categories as follows:

- Large Scientific Equipment represents more specialised and dedicated equipment for multi-user and inter-disciplinary research programmes. This sub-category of equipment refers to those pieces of equipment that are fundamental to undertaking competitive research, training postgraduate students and developing staff, particularly in terms of technical and applications expertise. The acquisition, development and upgrade of specialised equipment by a particular institution also requires that the research institution assume responsibility for the service and maintenance costs associated with large scientific equipment. The institution is further responsible for ensuring that the equipment is accessible to users from other institutions, including industry, at a fee that is based on a cost recovery charge-out rate (South African Department of Science and Technology, 2010). Examples of this class of equipment transmission and scanning electron microscopes.

- Advanced Scientific Equipment constitutes the acquisition or development of unique, state-of-the-art multi-user, inter-disciplinary and highly specialised scientific equipment that is not only able to push the frontiers of science, but is also able to address the development of scarce skills, attract industrial involvement, drive scientific and technological productivity and advance national priorities. In 
general, equipment in this sub-category is often too costly to be acquired by institutions individually and requires multi-institutional support. In the latter instance, institutions based within a specific geographical region tend to collaborate closely in order to either acquire or develop advanced scientific equipment that benefits the region itself. Depending on the scientific requirements, equipment of this nature may be placed in an independent location in a specific region in order to equally serve the needs of researchers within that region. In many instances this equipment will provide an international competitiveness to the development of a specific research area (South African Department of Science and Technology, 2010). An example extends to the suite of mass spectrometers available at the Institute of Wine Biotechnology at the University of Stellenbosch in the Western Cape. The institute focuses on understanding the biology of wine-associated organisms, including the ecology, physiology, molecular and cellular biology of grapevine, wine yeast and wine bacteria to promote the sustainable, environmentally friendly and cost-effective production of quality grapes and wine. The matrix-assisted laser desorption ionisation-time of flight mass spectrometry (MALDI-TOF MS) has aided the institute in the identification and diagnosis of microbes that contribute towards improving the quality of wine for both the local and global markets (Bauer, 2018).

Additional resources need to be earmarked to provide the necessary space, services, utilities, technical, operational maintenance, IT support, replacement and upgrade costs. In many cases, special attention to renovating physical infrastructures such as buildings may be required. An additional requirement motivating the investment in this sub-category of scientific equipment will be to establish a clear governance and/or management structure, and present a detailed business plan that clearly addresses the issue of sustainability.

The key criteria used for the provision of funding in the Scientific Equipment category may include:

- Equitable geographic distribution of equipment across the higher education sector including science councils in terms of access, areas of expertise and contribution to the national R\&D agenda.

- Demographic distribution in terms of allocating grants in line with the redress and equity targets of the country.

- Sustainable management of equipment in terms of its placement and efficient usage and maintenance.

- Social impact in terms of benefits derived from the placement of the equipment or infrastructure to the people and communities (South African Department of Science and Technology, 2010). 


\subsubsection{Specialised Facilities}

Specialised facilities (SF) are dedicated research performing institutions that houses large, unique and highly specialised physical RI that provides a controlled environment for ensuring the optimal performance of the research equipment as well as conducting highly specialised experiments. Examples of these types of research facilities include specially-constructed laboratories, biosafety containment laboratories, pre-clinical laboratories and research clean-rooms.

In the South African context, this category of RI includes the national research facilities (NFs). The NFs, managed by the NRF, play a critical role in the provision of unique and cutting-edge research infrastructure platforms in the country for the advancement of science and technology across the research enterprise. However, these facilities have been operating under financial duress, thereby constraining their ability to maintain and sustain the infrastructure platforms. This challenge has threatened the ability of the NFs to effectively deliver on their core mandates, i.e. accessibility, knowledge generation, human capital development, and science outreach and awareness.

National Facilities are centred on substantial instrumentation, equipment or skills base and is established to satisfy an identified national social, economic or technological need and which, because of expertise and capabilities, is justified on the basis of shared research and/or service used by external organisations. The facility is made available for research by internal and external researchers on the basis of the merit of proposals as assessed by peer-group review, while service work is commercially supplied to industry. The work programme of the facility is balanced to ensure an appropriate allocation of time to both research and service activities. (South African Department of Arts, Culture, Science and Technology, 1996)

In summary, advanced specialised laboratories refer to infrastructure platforms that not only include the physical laboratory in a specific location, but also the suite of highly specialised scientific equipment. In most instances the equipment and geographic location are integrally linked to form a single infrastructure platform, i.e. the equipment and experiments cannot function optimally unless the environment subscribes to specific physical and environmental standards such as appropriate air-conditioning, reinforced flooring, noise and vibration cancellation systems, as well as controlled environments for humidity and temperature. Some examples of such laboratories include specialised microscopy facilities, such as the high resolution microscopy facilities; bio-repositories; radio-telescopes; research-focused forensic laboratories; research museums; research clean-rooms; biosafety, biohazard and radiation containment facilities; and oceanographic facilities. 
The key elements linked to the provision of funding in this category include the:

- Physical infrastructure that is required to house the research equipment;

- Ancillary equipment or feeder equipment that will complement the capabilities of the research equipment;

- Research equipment;

- Funding towards the operational and maintenance costs as well as technical support that are required to ensure the sustainable management of the specialised laboratories.

The NFs, as outlined in Table 2.1 summarise South Africa's investment in this category of RI. Unfortunately these NFs have been operating under financial duress, which has consequently lead to constraining their ability to maintain and sustain the infrastructure platforms. This challenge has threatened the ability of the NFs to effectively deliver on their core mandates, i.e. accessibility, knowledge generation, human capital development, and science outreach and awareness. Some of the challenges include the inability to:

- Renew ageing equipment and infrastructure;

- Succeed and replace the aging workforce;

- Effectively manage ageing and obsolete equipment and infrastructure so as to minimise disruptions in operations;

- Acquire necessary state-of-the-art equipment to meet commitments and mandates; and

- Maintain and acquire additional infrastructure, including the upgrade of the existing infrastructure, to keep up with advancing technological developments (South African Department of Science and Technology, 2010).

In attempting to address some of the challenges reported above, the SARIR which was launched in 2016 attempts to prioritise the investment in the establishment of 13 specialised facilities across five thematic areas, as described in Table 1.1.

\subsubsection{High-End Infrastructure}

High-end infrastructure refers to the infrastructure needed to bridge the "innovation chasm" (refer to Sect. 1.2) with the objective of strengthening the commercialisation potential of products, processes and services. High-end infrastructure refers to specialised platforms or laboratories that support the transition from R\&D to commercialisation. This type of infrastructure is required to demonstrate scalability and reproducibility in terms of processes, quantities and quality which are necessary prerequisites to full-scale manufacturing and commercialisation. It is also a crucial and necessary step to mitigate risk and secure venture capital (South African Department of Science and Technology, 2010). Examples of RI in this category include pilot plants, incubators, technology demonstrators and semi-commercial test facilities (Fig. 2.4). 
Table 2.1 A summary of the NRF-managed national research facilities

\begin{tabular}{|c|c|c|}
\hline National facility & Core business & $\begin{array}{l}\text { Grand challenge that is } \\
\text { addressed }\end{array}$ \\
\hline $\begin{array}{l}\text { The South African Radio } \\
\text { Astronomy Observatory } \\
\text { (SARAO) }\end{array}$ & $\begin{array}{l}\text { SARAO supports research } \\
\text { and training in radio } \\
\text { astronomy and space } \\
\text { geodesy. It includes the (i) } \\
\text { Square Kilometre Array } \\
\text { Radio Telescope, commonly } \\
\text { known as the SKA; (ii) radio } \\
\text { astronomy instruments and } \\
\text { programmes such as the } \\
\text { MeerKAT and KAT-7 } \\
\text { telescopes in the Karoo; (iii) } \\
\text { the Hartebeesthoek Radio } \\
\text { Astronomy Observatory } \\
\text { (HartRAO); and (iv) the } \\
\text { African Very Long Baseline } \\
\text { Interferometry (AVN) } \\
\text { programme in nine African } \\
\text { countries }\end{array}$ & $\begin{array}{l}\text { Space Science and } \\
\text { Technology }\end{array}$ \\
\hline $\begin{array}{l}\text { iThemba Laboratory for } \\
\text { Accelerator Based Sciences } \\
\text { (iThemba LABS) }\end{array}$ & $\begin{array}{l}\text { Provides advanced, viable, } \\
\text { multi-disciplinary facilities } \\
\text { for training and services in } \\
\text { the fields of sub-atomic } \\
\text { nuclear sciences and applied } \\
\text { radiation medicine }\end{array}$ & $\begin{array}{l}\text { Farm to pharma (radiation } \\
\text { therapy and isotope } \\
\text { production) } \\
\text { Energy security (High-energy } \\
\text { nuclear physics) }\end{array}$ \\
\hline $\begin{array}{l}\text { South African Astronomical } \\
\text { Observatory (SAAO) }\end{array}$ & $\begin{array}{l}\text { Performs fundamental } \\
\text { research in astronomy and } \\
\text { astrophysics and supports the } \\
\text { Southern Africa Large } \\
\text { Telescope (SALT) }\end{array}$ & $\begin{array}{l}\text { Space Science and } \\
\text { Technology }\end{array}$ \\
\hline $\begin{array}{l}\text { South African Environmental } \\
\text { Observation Network } \\
\text { (SAEON) }\end{array}$ & $\begin{array}{l}\text { An emerging facility that } \\
\text { establishes and maintains } \\
\text { nodes linked by an } \\
\text { information management } \\
\text { network. These nodes serve } \\
\text { as research and education } \\
\text { platforms for long-term } \\
\text { studies of ecosystems and } \\
\text { assist in the development of } \\
\text { methods to detect, predict and } \\
\text { react to environmental change }\end{array}$ & $\begin{array}{l}\text { Global Change (long term } \\
\text { in situ environmental } \\
\text { monitoring, environmental } \\
\text { information systems) }\end{array}$ \\
\hline $\begin{array}{l}\text { South African Institute for } \\
\text { Aquatic Biodiversity } \\
\text { (SAIAB) }\end{array}$ & $\begin{array}{l}\text { Serves as a research hub for } \\
\text { aquatic biodiversity in } \\
\text { Southern Africa by housing } \\
\text { and developing the National } \\
\text { Fish Collection as well as } \\
\text { other sources of aquatic } \\
\text { biodiversity data }\end{array}$ & $\begin{array}{l}\text { Global Change (long term } \\
\text { observation of biodiversity in } \\
\text { aquatic environment) }\end{array}$ \\
\hline
\end{tabular}




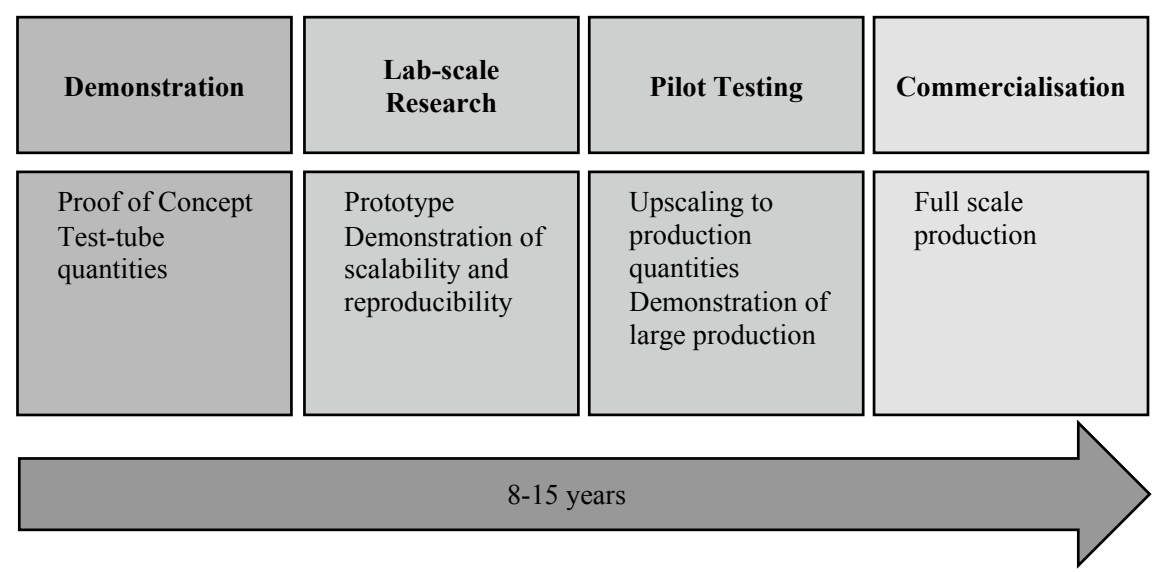

Fig. 2.4 Key components of product development within the innovation value chain (South African Department of Science and Technology, 2010)

The key objectives for building, operating and maintaining pilot plants are as follows:

- To reduce the technical and financial risks for scaling up the selected technology to full scale production;

- To reduce marketing risks by producing sufficient quantities of the product that can be tested by potential customers;

- To troubleshoot, align and resolve any challenges that may impact on the downstream processing technologies required for full scale production;

- To provide an experiential training facility for future employees of the envisaged full-scale plant; and

- To provide a facility for ongoing technology development such that there exists the possibility to expand the proposed range of products and/or services (South African Department of Science and Technology, 2010).

The key criteria for the provision of RI funding in this category may therefore include assessing the level of: (i) innovation; (ii) economic impact; (iii) industry partnerships; and (iv) beneficiation of raw materials.

\subsubsection{Global Research Infrastructures}

Global research infrastructures (GRIs) are recognised as critical enablers for advancing scientific knowledge, research outputs and innovations, as well as accelerating the training and development of the next generation researchers (Group of Senior Officials, 2017). Global research infrastructures can be classified as (i) 'single-sited', i.e. a single resource at a single location, such as the Large Hadron Collider (LHC); 
(ii) 'distributed', i.e. being part of a network of distributed resources, such as ocean, earth or seafloor observatories; or (iii) 'virtual', i.e. the service is provided remotely, such as simulation environments. Regardless of the type of global infrastructure, there is a fundamental need for the management of big data and high-speed networks for the optimal sharing of data and other resources (South African Department of Science and Technology, 2010).

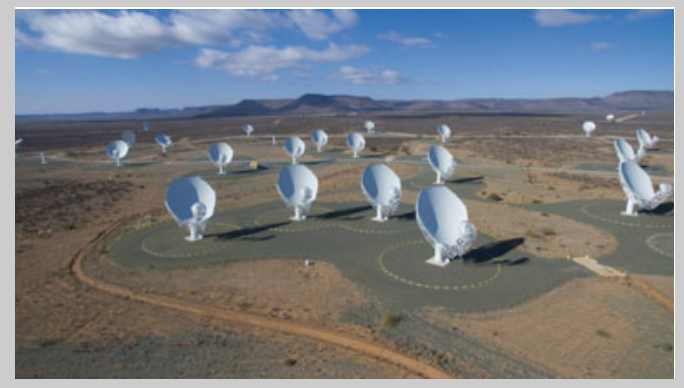

The SKA project is an international effort to build the world's largest radio telescope, with eventually over a square kilometre (one million square metres) of collecting area. It is one of the largest scientific endeavours in history and brings together scientists, engineers and policy makers from around the world. The SKA core high and mid frequency telescopes are hosted in South Africa's Karoo region, ultimately extending over the African continent. Australia's Murchison Shire hosts the project's low-frequency antennas.

In this regard, GRI facilities are critical enablers for high quality teaching and training as well as conducting cutting edge research and driving innovation. This category of funding requires parallel investments in travel or mobility grants to facilitate access to RI facilities, including the outbound access to GRIs, which refers to national researchers travelling abroad, usually to the global north, to GRIs such as synchrotrons and the LHC at the Conseil Européen pour la Recherche Nucléaire (CERN), to name a few. The other type of access is inbound access, which refers to researchers accessing those GRIs that are located within a specific country. In the South African context, these include the national research facilities: Southern African Large Telescope (SALT) and Square Kilometre Array (SKA).

As seen by the examples presented above, the complexity and high development, construction and operational costs associated with GRIs make it rather difficult for a single country to build, maintain and operate. Consequently, efforts towards the internationalisation of large-scale GRI that have evolved to meet the scientific demands that extend further than the geographical boundaries of individual countries or institutions (Group of Senior Officials, 2017). It requires a multi-pronged and multi-user 
approach in terms of leadership, scope, cost and complexity. Global research infrastructures have been identified as research platforms that do not only provide essential RI platforms for the generation of internationally competitive science and technology outputs but also represent global collaborative geared towards addressing key sustainable development challenges that are articulated in the SDGs and STISA. These GRIs are influential in attracting the best researchers from around the world and building bridges between national and international research communities and scientific disciplines to address research issues that cannot be tackled by a single institution, region or country services (South African Department of Science and Technology, 2010).

The potential for increased cooperation on GRIs has long been recognised at high-level science diplomacy meetings. A Group of Senior Officials (GSO) on GRI was established at the first Group of Eight (G8) ${ }^{1}$ Science Ministers' Meeting in 2008 (European Commission, 2018) and has been active since 2011. The primary objectives of the GSO are to: (i) identify RI of global interest, (ii) analyse how countries evaluate and prioritise the construction of large scale RIs, (iii) identify possible new areas of cooperation, (iv) promote transnational access to GRIs, (v) foster "distributed" RIs, (vii) identify measures to ensure that scientific data is appropriately handled, stored and accessed, and (vi) adopt a common understanding for the joint lifecycle management of GRIs (European Commission, 2018).

The GSO advocates for global excellence-driven access to the GRI. The recommendation by the GSO to GRIs is to employ peer review processes that approves access based on scientific excellence of the most promising emergent ideas, regardless of the country's membership status with the GRI (Group of Senior Officials, 2017).

Membership tends to be defined in medium to long term contractual arrangements between GRIs and countries. When a country enters into a fixed-term (or indefinite) membership agreement with a GRI, dedicated funding is required by that country for membership fees that facilitate the access of researchers in that country, to the GRI. Funding directed towards supporting this venture requires due consideration to the following costs:

- Membership fees, including contributions towards the maintenance and upgrade of the infrastructures at the GRI;

- Mobility and other related travel;

- Accommodation; and

- Charge-out fees.

The processes employed by GRIs to allocate access time to utilise their infrastructure facilities to both member and non-member countries is essentially based on a merit system, underpinned by scientific excellence (Group of Senior Officials, 2017). In general, the following process is adopted by GRIs:

\footnotetext{
${ }^{1}$ GSO is composed of representatives from Australia, Brazil, Canada, China, the European Commission, France, Germany, India, Italy, Japan, Mexico, Russia, South Africa, United Kingdom and United States of America (European Commission, 2018).
} 
- Calls for proposals are opened by the GRI;

- Researchers across the globe apply through the GRI application process to utilise the facilities/laboratories/beamlines;

- Discipline specific peer review processes are undertaken by the GRI. All applications are reviewed against excellence; and

- Outcomes of the review process are communicated to all applicants by the GRI. Successful applicants are provided with the contact details of the manager of each facility/laboratory/beamlines, to proceed to book access time.

A common approach used by most GRIs is to allocate a set portion, of the total available access time to usage by the researchers from countries that do not hold membership, but have demonstrated excellence in their applications.

Some of the key international GRI facilities that are currently accessed by South African scientists, through formal collaborative agreements, is summarised in Table 2.2. The underpinning objectives linked to the access to GRIs include:

- To grow the expertise pool, in terms of (i) postgraduate students; (ii) emerging researchers; and (iii) staff development, with respect to building their research

Table 2.2 Summary of current and proposed SA memberships to GRIs (National Research Foundation, 2018)

\begin{tabular}{l|l}
\hline Name of GRI & Objectives \\
\hline Joint Institute for Nuclear Research (JINR) & $\begin{array}{l}\text { The main objectives for South Africa's } \\
\text { membership to JINR is to provide the South } \\
\text { African research community with access to } \\
\text { world-class facilities, research and networking } \\
\text { opportunities in nuclear sciences with the } \\
\text { JINR scientific community in Dubna, Russia }\end{array}$ \\
\hline $\begin{array}{l}\text { European Synchrotron Radiation Facility } \\
\text { (ESRF) }\end{array}$ & $\begin{array}{l}\text { The main objectives for South Africa's } \\
\text { membership to ESRF is to provide the South } \\
\text { African research community with access to } \\
\text { world-class synchrotron facilities, including } \\
\text { the various beamlines as well as research and } \\
\text { networking opportunities across a multitude } \\
\text { of research disciplines }\end{array}$ \\
\hline European Organisation for Nuclear Research \\
(CERN) & $\begin{array}{l}\text { The SA-CERN programme gives South } \\
\text { African researchers and postgraduate students } \\
\text { access to the largest open research facility in } \\
\text { the world, which is based in Switzerland. } \\
\text { South African researchers and postgraduate } \\
\text { students participate in a SA-CERN Theory } \\
\text { Group and in three experiments in the Large } \\
\text { Hadron Collider (LHC) at CERN, viz } \\
\bullet \text { ATLAS (a Torodial LHC Apparatus); } \\
\bullet \text { ALICE (a Large Ion Collider Experiment); } \\
\text { and } \\
\bullet \text { ISOLDE (Isotope Separator on Line Device) }\end{array}$ \\
\hline
\end{tabular}


capacity and increasing knowledge outputs in specialised research areas that align to the geographical positioning of the country;

- To stimulate the development of technical expertise and technology transfer through both outbound and inbound collaborations;

- To build a strong local infrastructure base that complements the capabilities of the instrumentation available at GRIs. This allows for optimal and effective usage of the instrumentation at GRIs by national researchers; and

- To foster and nurture international partnerships that advance the science trajectory of the country.

Besides the facilitation of joint research, innovation and knowledge sharing, GRIs play a key in the training of students and researchers. The teaching and training programmes for researchers and students at the local infrastructure facility may include joint hosting of (i) winter or summer schools; (ii) specialist schools; and (iii) reciprocal collaborative support programmes that encourage researchers and students based at international GRI facilities to visit the local infrastructure base. These interventions are necessary to enable the scientists and students to derive maximum benefit from participation in the projects undertaken at global infrastructure facilities. It also strengthens synergies of scientific endeavours on the continent which, at a later stage, can be leveraged to consider the establishment of a singular African memberships to GRIs that are hosted in the global north. The partnering of countries on the continent will also lead to a more sustainable and cost effective mechanism for accessing essential GRI platforms.

Over and above the formal agreements with GRIs, a country should have in place a general equipment-related travel and training grant that makes funds available to support the larger science community with the objective of affording access to (i) other internationally based state-of-the-art equipment that does not form part of any formal agreements; and (ii) nationally-based research equipment that is not available at the home research institution or region.

\subsubsection{Cyber-Infrastructure}

Cyber-infrastructure refers to information and communication technology (ICT)based infrastructures such as (i) high performance computing; (ii) broadband research networks; (iii) data storage and management systems; and (iv) grid and cloud computing infrastructures. These platforms contribute to the comprehensive infrastructure that is needed to address the complex, multi-disciplinary and cross-border needs of modern science. The evolution of science and technology has relied heavily on the exploitation of advances in ICT and the integration of hardware for computing, data management and manipulation as well as experimental facilities that require an inter-operable suite of software and middleware services and tools (South African Department of Science and Technology, 2010). 
Investments in cyber-infrastructure is driven by the ever increasing need for analysis and storage of large data sets from many sources, including data captured by research equipment, data generation by simulations and sensor networks. Examples of disciplines that are mostly affected by such large data-sets include genomics and astronomy. These demands have given rise to e-science, which can be defined as the set of tools and technologies that support data federation and collaboration for the purposes of data analysis and mining, data visualisation and exploration, and communication. As a consequence, there are growing investments in e-infrastructure which refers to a combination and interworking of (i) digitally-based technology (hardware and software); (ii) resources such as data, services and digital libraries; (iii) communication, which includes protocols, access rights and networks; and (iv) people needed to support modern, internationally leading collaborative research across the sciences (Hey, Tansley, \& Tolle, 2009).

The investments in this category of funding links to (i) high bandwidth networks; (ii) infrastructure; (iii) open-source; (iv) technologies and standards for data provenance, curation and preservation; (v) super-computing; and (vi) training of scientific software engineers and data scientists (Hey et al., 2009).

Cyberinfrastructure consists of computing systems, data storage systems, advanced instruments and data repositories, visualisation environments, and people, all linked together by software and high performance networks to improve research productivity and enable breakthroughs not possible otherwise. (Steward, Simms and Plale, 2010)

Cyber-infrastructure plays a critical role in the knowledge-triangle (Fig. 1.1) as well as the innovation value chain. Cyber-infrastructure underpins the various categories of research infrastructure as proposed in this book, which include (i) scientific equipment; (ii) specialised facilities; (iii) high-end infrastructure; and (iv) GRIs. This type of infrastructure essentially requires computing, data storage and management, transmission and/or communication networks and application development services. Cyber-infrastructure is a pre-requisite in addressing pertinent issues such as the need to store, analyse and process unprecedented amounts of heterogeneous data and information that form the enabling backbone that supports the establishment of world-class scientific collaborations as well as accessing and sharing scientific resources and information regardless of the source or nature of such information or its location. Closely linked to enhanced computational power and networks as well as data storage and management is the need for applications and competence development that focuses on the establishment, optimal use and sustainability of cyber-infrastructure in South Africa (South African Department of Science and Technology, 2010).

The South African Information and Communication Technology Research Development and Innovation (ICT-RDI) Roadmap was launched in 2015. This roadmap provides strategic national direction, a set of action-plans and an implementation 
framework to guide, plan, co-ordinate and manage South Africa's investment in the sector (South African Department of Science and Technology, 2015). This framework highlights six clusters of opportunity, such as:

- Broadband services and infrastructure, which relates to both future wireless technologies and broadband service infrastructure. While wireless technologies relate to the design and development of technologies that respond to changes in the market-demand for wireless broadband services, broadband service infrastructure focuses on the utilisation of public broadcast and wireless spectrums with the intention to increase access via more available and less costly broadband.

- ICT for development refers to the application of ICT that contributes towards socioeconomic impact. This includes (i) enhancing agricultural production; and (ii) the promoting e-inclusion for the removal of barriers to the use of ICT technologies by disadvantaged individuals and communities.

- Sustainability and the environment refers to using ICT to (i) support a greener environment; (ii) sense, observe and model global changes relating to climate, human migration, and environmental factors to name a few; and (iii) geo-spatial applications relating to observations from space and in situ environmental and disaster management.

- Industry applications makes reference to smart infrastructures, mining, manufacturing, future internet applications, content creation and delivery, supply chain optimisaation and asset management.

- Grand sciences, includes (i) the big science initiatives in the country, such as the SKA initiative, to aid in data gathering, filtering, storage and mining techniques; and (ii) the application of ICT to bio-medical sciences especially in the area where biology meets medicine.

- Service economy includes the usage of ICT in mobile health, e-services, education, business model innovation and payment solutions (South African Department of Science and Technology, 2015).

These six clusters are influenced by big data, which is structurally diverse, complex and dynamic in nature (refer to Sect. 7.5), thereby posing a problem in many areas of science including innovation, technology, engineering, social sciences, arts and humanities (Fig. 2.5). The main components of the current South African cyberinfrastructure framework involves the National Integrated Cyber-infrastructure System (NICIS), which includes the Centre for High Performance Computing (CHPC), South African National Research Network (SANReN), the Data Intensive Research Initiative of South Africa (DIRISA) as well as the South Africa GRID Computing (SA-GRID) and the Cloud Initiative, which is still in the conceptual phase. 


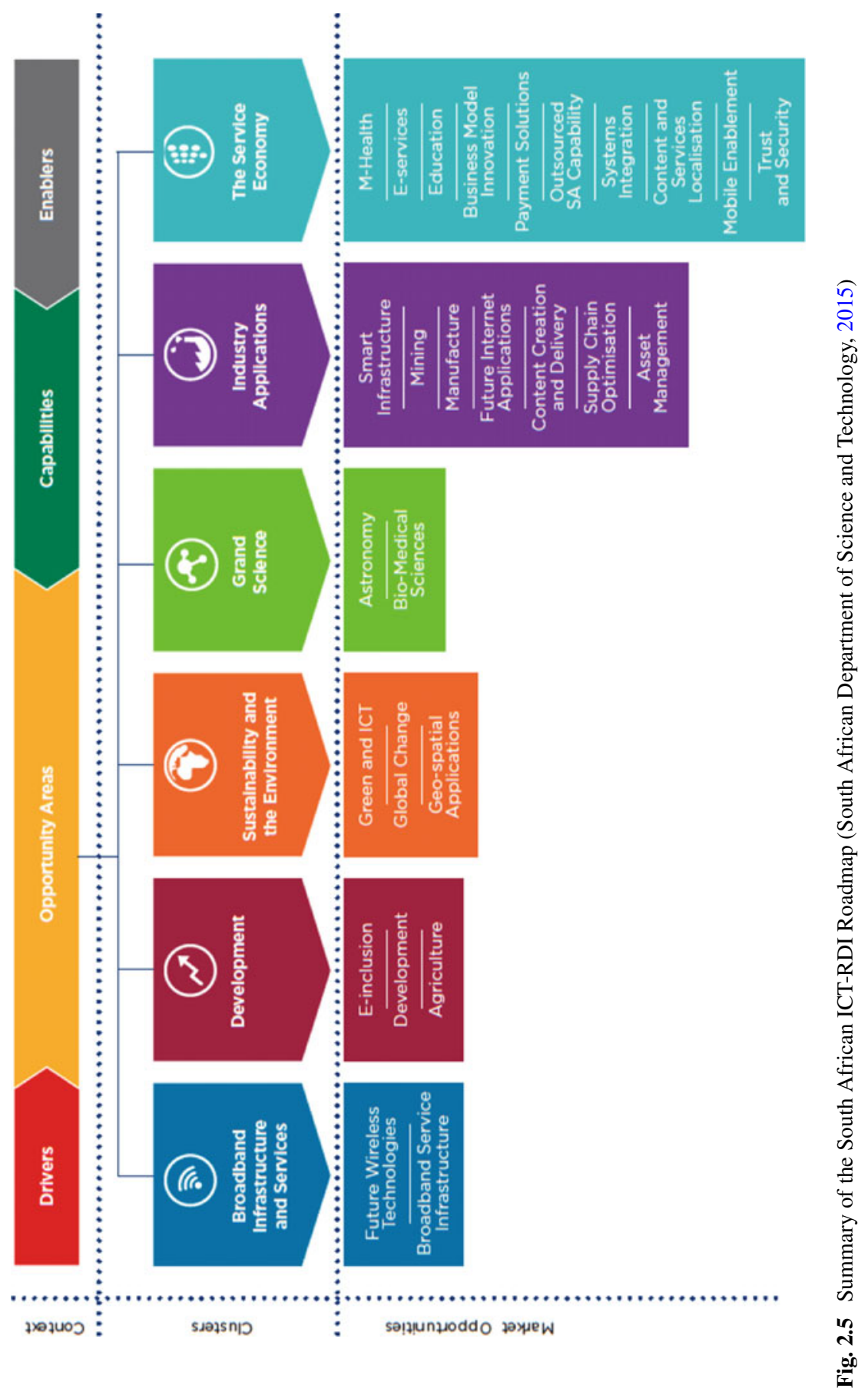




\subsection{Summary}

It is evident that the successful implementation of STI strategies and policies is heavily reliant on the provision of a strong RI base, a skilled workforce, financial resources and collaborative networks. Research infrastructure can stimulate innovative research across the innovation value chain, critical for the realisation of a vibrant national system of innovation. The classification system presented here highlights the important role of research infrastructure in the advancement of (i) science, technology and innovation efforts; and (ii) skills development in the country.

In order to inspire the research enterprise to develop world class leaders, it is necessary to invest in RI, scientific equipment and specialised laboratories to ensure that the objectives set forth by the national policies are addressed at the upstream end of the innovation value chain. For instance, in order to bridge the gap between knowledge generation and the realisation of the commercial potential associated with the application of that knowledge, it is important to invest in high-end infrastructure. Furthermore, cyber-infrastructure forms the foundation for various RI which essentially require computing, data storage and management, transmission and/or communication networks and application development services. Cyber-infrastructure is a prerequisite in addressing issues such as the need to store, analyse and process unprecedented amounts of heterogeneous data and information. It forms the enabling backbone towards accessing and sharing scientific resources and information regardless of the nature of such information and its location.

Finally, the support of 'Fundamental or Basic Science' questions is an area of priority investment for any country. Hence it is important to provide mechanisms that facilitate mobility and access to these global infrastructures, necessary for the development and advancement of research capacity and human capital in the quest to seek answers to questions that relate to understanding the building blocks of life or global challenges such as climate change, biodiversity, energy security, health, food and water security.

\section{References}

Bauer, F. (2018). Research programmes in wine- and grapevine biotechnology. Available at: https://www.sun.ac.za/english/faculty/agri/wine-biotech/research/research-programmesin-wine-biotechnology. Accessed October 31, 2018.

European Commission. (2018). Group of senior officials on global research infrastructures. Available at: https://ec.europa.eu/info/research-and-innovation/strategy/europeanresearch-infrastructures/group-senior-officials-gso_en. Accessed March 1, 2019.

Girdwood, J. (2013). Top-down and bottom-up approaches within implementation. Available at: https://politicalpipeline.wordpress.com/2013/02/21/top-down-and-bottom-up-approacheswithin-implementation/. Accessed October 9, 2018.

Group of Senior Officials on Global Research Infastructures. (2017). GSO Framework. Available at: https://ec.europa.eu/research/infrastructures/pdf/gso_framework_for_global_ris. pdf. Accessed April 14, 2019. 
Hey, T., Tansley, S., \& Tolle, K. (2009). The fourth paradigm: Data-intensive scientific discovery. Washington: Microsoft Corporation.

Khadka, C., \& Vacik, H. (2012). Comparing a top-down and bottom-up approach in the identification of criteria and indicators for sustainable community forest management in Nepal. Forestry: An International Journal of Forest Research, 85(1), 145-158.

National Research Foundation. (2018). Infrastructure funding instrument: National equipment programme framework and funding guide. Available at: http://www.nrf.ac.za/sites/default/ files/documents/NEP\%20Call\%20Framework\%20and\%20Funding\%20Guide\%202019.pdf.

Accessed September 21, 2018.

Neethling, J. H. (2018). Advisory board meeting: 10 May 2018. South Africa: Nelson Mandela University.

South African Department of Arts, Culture, Science and Technology. (1996). White paper on science and technology: Preparing for the 21st century. Available at: http://www.esastap.org.za/ download/st_whitepaper_sep1996.pdf. Accessed October 2, 2017.

South African Department of Science and Technology. (2002). National research and development strategy. Available at: http://www.esastap.org.za/download/sa_natrdstrat_aug2002.pdf. Accessed January 12, 2017.

South African Department of Science and Technology. (2010). Research, development and innovation funding framework. Available at: https://notices.ukzn.ac.za/content/GetFile.aspx?id=1424. Accessed January 12, 2016.

South African Department of Science and Technology. (2015). Information and communication technology research, development and innovation roadmap. Available at: http://www.dst.gov.za/ images/ict_rdi_roadmap.pdf. Accessed September 23, 2017.

Stewart, C. A., Simms, S., Plale, B., Link, M., Hancock, D. Y., \& Fox, G. C. (2010). What is Cyberinfrastructure? Proceedings ACM SIGUCCS User Services Conference. https://doi.org/ $10.1145 / 1878335.1878347$.

Open Access This chapter is licensed under the terms of the Creative Commons Attribution 4.0 International License (http://creativecommons.org/licenses/by/4.0/), which permits use, sharing, adaptation, distribution and reproduction in any medium or format, as long as you give appropriate credit to the original author(s) and the source, provide a link to the Creative Commons license and indicate if changes were made.

The images or other third party material in this chapter are included in the chapter's Creative Commons license, unless indicated otherwise in a credit line to the material. If material is not included in the chapter's Creative Commons license and your intended use is not permitted by statutory regulation or exceeds the permitted use, you will need to obtain permission directly from the copyright holder.

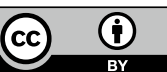

